\title{
Cajanine promotes osteogenic differentiation and proliferation of human bone marrow mesenchymal stem cells
}

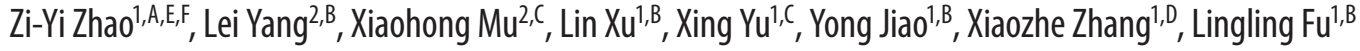 \\ ${ }^{1}$ Department of Orthopedics, Dongzhimen Hospital, Beijing University of Chinese Medicine, China \\ ${ }^{2}$ Cancer Center, ${ }^{\text {st }}$ Hospital of Jilin University, Changchun, China \\ A - research concept and design; $\mathrm{B}$ - collection and/or assembly of data; $\mathrm{C}$ - data analysis and interpretation; \\ $D$ - writing the article; $E$ - critical revision of the article; $F$ - final approval of the article
}

\section{Address for correspondence}

Zi-Yizhao

E-mail:drzhaoziyi@sina.com

\section{Funding sources}

This work was supported by the National Natural Science Foundation of China (30600847).

Conflict of interest

None declared

Received on June 14, 2016

Reviewed on June 29, 2017

Accepted on August 24, 2017

Published online on August 24, 2018

Cite as

Zhao Z-Y, Yang L, MuX, et al. Cajanine promotes osteogenic differentiation and proliferation of human bone marrow mesenchymal stem cells. Adv Clin Exp Med. 2019;28(1):45-50. doi:10.17219/acem/76638

DOI

10.17219/acem/76638

\section{Copyright}

Copyright by Author(s)

This is an article distributed under the terms of the

Creative Commons Attribution Non-Commercial License

(http://creativecommons.org/licenses/by-nc-nd/4.0/)

\begin{abstract}
Background. Seed cells - mesenchymal stem cells (MSCS) - appear to be an attractive tool in the context of tissue engineering. Bone marrow represents the main source of MSCs for both experimental and clinical studies. However, the number limitation of bone marrow MSCS (BMSCS) and decreased function caused by proliferation make the search for adequate alternative sources of these cells for autologous and allogenic transplant necessary.
\end{abstract}

Objectives. This study was aimed to investigate the roles of cajanine isolated from the extracts of Cajanus cajan L. Millsp. in the proliferation and differentiation of BMSCs, and to discover the mechanism of proliferation of BMSCs promoted by cajanine.

Material and methods. Bone marrow mesenchymal stem cells were cultured in high-glucose Dulbecco's Modified Eagle's Medium (DMEM) and osteogenic differentiation was induced by adding dexamethasone, ascorbic acid and $\beta$-glycerophosphate supplements. Bone marrow MSCs were cultured in medium without cajanine or supplemented with cajanine. The information about the proliferation and osteogenic differentiation of BMSCs was collated. The osteogenic differentiation potential of BMSCs was also assessed at the $3^{\text {rd }}$ passage by Von Kossa staining. To observe cell signal transduction changes of BMSCs after culturing them with cajanine for $24 \mathrm{~h}$, the western blot analysis was performed to detect phosphorylated cell cycle proteins and activated cyclins.

Results. After osteogenic induction, the differentiation of BMSCs was accelerated by cajanine treatment. Osteogenesis markers were upregulated by cajanine treatment at both protein and mRNA levels. Cajanine obviously promoted the proliferation of BMSCS. After BMSCs were cultured with cajanine for $24 \mathrm{~h}$, the cell cycle regulator proteins were phosphorylated or upregulated.

Conclusions. Cajanine can promote the expansion efficiency of BMSCs, at the same time keeping their multi-differentiation potential. Cajanine can activate the cell cycle signal transduction pathway, thus inducing cells to enter the $\mathrm{G} 1 / \mathrm{S}$ phase and accelerating cells entering the $\mathrm{G} 2 / \mathrm{M}$ phase. This study can contribute to the development of cajanine-based drugs in tissue engineering.

Key words: mesenchymal stem cells, placenta, Cajanus cajan L., tissue engineering 


\section{Introduction}

Cajanus cajan (cajanine) is a perennial legume from the family Fabaceae. ${ }^{1}$ It is extracted from pigeon pea leaves and has been reported to exhibit a variety of bioactivities, including antimalaria, hypolipidemia and cytotoxicity effects, as well as reducing bone loss and promoting bone-like tissue formation. ${ }^{1-7}$ Moreover, a recent study revealed that cajanine could increase tibial bone density and improve bone metabolism as well as lipid metabolism in ob/ob mice with osteoporosis and hyperlipidemia symptoms, indicating that cajanine might be a promising drug candidate due to its broad range of bioactivities. ${ }^{8}$

Mesenchymal stem cells (MSCs) are a small population of multipotent stromal cells that are present in practically all tissues. ${ }^{9}$ Mesenchymal stem cells are maintained in a relative state of quiescence in vivo; however, they are capable of proliferating and then differentiating into osteoblasts, chondrocytes, adipocytes, or other mesoderm-type lineages when stimulated by a variety of physiological and pathological signals. ${ }^{9}$ Multiple signaling networks have been demonstrated for the development and differentiation of MSCs into functional mesenchymal lineages. The transforming growth factor- $\beta$ (TGF- $\beta$ ) signal pathway has been studied as a key regulator in the self-renewal, maintenance and differentiation of stem cells. ${ }^{10-12}$ It has been reported that TGF- $\beta 1$ plays an important role in cartilage development and is an important chondrogenic factor. ${ }^{12,13}$ Moreover, some studies have demonstrated that cajanine has anti-tumor and anti-oxidant functions. ${ }^{14}$ Despite evidence revealing that cajanine has potential to be a therapeutic agent, the detailed mechanisms of anti-tumor effects are still under investigation.

In this study, the roles of cajanine in the proliferation and differentiation of bone marrow MSCs (BMSCs) were investigated. The osteogenesis markers and cell cycle were further assessed.

\section{Material and methods}

\section{Bone marrow mesenchymal stem cells culture}

Human bone marrow aspirates were obtained from donors from Dongzhimen Hospital, Beijing, China, in the years 2012-2014, and isolated by density gradient centrifugation, utilizing Ficoll-Paque TM PLUS solution (GE Healthcare, Uppsala, Sweden), followed by cell-surface marker negative selection according to the previous report. ${ }^{15}$ Bone marrow MSCs isolated from donors were used within 4 passages. Briefly, cells were harvested using 0.25\% trypsin with 1.0 M ethylenediaminetetraacetic acid (EDTA), centrifuged and expanded in basal medium, consisting of high-glucose Dulbecco's Modified Eagle's Medium (DMEM), supplemented with $10 \%$ fetal bovine serum (FBS),
$100 \mathrm{unit} / \mathrm{mL}$ penicillin and $100 \mathrm{unit} / \mathrm{mL}$ streptomycin (basal medium). Basal medium contained high-glucose DMEM, FBS, penicillin and streptomycin (all from Gibco, Gaithersburg, USA). Cells were maintained in a $37^{\circ} \mathrm{C}$ incubator. The medium was changed every 3 days.

\section{Chemicals and antibodies}

Cajanine was obtained from Sigma-Aldrich (Shanghai, China). Rabbit anti-collagen I polyclonal antibody was purchased from Abcam (ab21286; Abcam, Cambridge, UK); rabbit anti-osteopontin polyclonal antibody was purchased from Abcam (ab8448); rabbit anti-Runx2 monoclonal antibody was purchased from Cell Signaling (No. 12556; Beverly, USA); phospho-Rb rabbit monoclonal antibody was purchased from Cell Signaling (No. 8516); rabbit antiCDK2 monoclonal antibody was purchased from Cell Signaling (No. 2546); rabbit anti-CDC25A and anti-CDC25B monoclonal antibodies were purchased from Cell Signaling (No. 3652, No. 9525); rabbit anti-cyclin B1 monoclonal antibody was purchased from Cell Signaling (No. 12231); and rabbit anti- $\alpha$-tubulin monoclonal antibody was purchased from Cell Signaling (No. 2144).

\section{Osteogenic induction}

Osteogenic differentiation was performed by culturing $3 \times 10^{4}$ human BMSCs (hBMSCs) in a 6-well plate in Poietics ${ }^{\circledR}$ osteogenic induction medium (Lonza Walkersville, Inc., Walkersville, USA), which contained dexamethasone, ascorbic acid and $\beta$-glycerophosphate supplements.

\section{Von Kossa staining}

The cells were fixed in cold methanol for 15-20 min at $-20^{\circ} \mathrm{C}$, followed by incubation with $5 \%$ silver nitrate solution (ab150687; Abcam), and crosslinked under UV light for 20-30 $\mathrm{min}$, then rinsed twice with distilled water. The dishes were incubated with $5 \%$ sodium thiosulfate solution for 2-3 min. After rinsing twice, the dishes were finally incubated with nuclear fast red solution for $5 \mathrm{~min}$, and rinsed with distilled water to remove excess stain.

\section{Real-time polymerase chain reaction}

Total RNAs were extracted using the TRIzol ${ }^{\circledR}$ reagent (Sigma-Aldrich), according to the manufacturer's instructions. After diminishing genomic DNA contamination by Turbo DNAse (Thermo Fisher Scientific, Waltham, USA), according to the manufacturer's instructions, the purified RNA $(10 \mathrm{ng} / \mathrm{mL}$ ) was reverse-transcribed with the High Capacity cDNA Reverse Transcription (RT) Kit (Qiagen, Valencia, USA) under the following conditions: $25^{\circ} \mathrm{C}$ for $10 \mathrm{~min}, 37^{\circ} \mathrm{C}$ for $120 \mathrm{~min}$, followed by $85^{\circ} \mathrm{C}$ for $5 \mathrm{~min}$. The real-time polymerase chain reaction (RT-PCR) was performed on an Applied Biosystems StepOnePlus ${ }^{\mathrm{TM}}$ PCR 
machine (Applied Biosystems, Foster City, USA), using $5 \mu \mathrm{L}$ SYBR ${ }^{\circledR}$ Green PCR Master Mix (Life Technologies, Grand Island, USA), $2 \mu \mathrm{L}$ sequence specific primers $(0.5 \mathrm{mM}$; glyceraldehyde-3-phosphate dehydrogenase (GAPDH) was used at the amount of $0.25 \mathrm{mM}$ ) and $3 \mu \mathrm{L}$ cDNA under the following conditions: $95^{\circ} \mathrm{C}$ for $10 \mathrm{~min}$, followed by 40 cycles of $15 \mathrm{~s}$ of denaturation at $95^{\circ} \mathrm{C}$ and $60 \mathrm{~s}$ of annealing and elongation at $60^{\circ} \mathrm{C}$. A melting curve analysis was performed after each run to confirm the product specificity. The $2 \Delta \mathrm{Ct}$ method was employed to determine the relative gene expression level of the gene of interest, normalized to the endogenous controls. ${ }^{5}$

\section{Cell proliferation assays}

Cell proliferation rates were measured by a methylthiazol tetrazolium (MTT) assay, using Cell Proliferation Assay Kit (Thermo Fisher). After the cells were treated with cajanine, MTT was added to each well and the cells were incubated at $37^{\circ} \mathrm{C}$ for $2 \mathrm{~h}$. The plates were centrifuged at $450 \times \mathrm{g}$ for $5 \mathrm{~min}$ at room temperature and the medium was removed. To solubilize the crystals, dimethyl sulfoxide (DMSO) was added to each well and the plates were read at $570 \mathrm{~nm}$, using a microplate reader (Molecular Devices, Sunnyvale, USA). The same experiment was repeated 3 times.

\section{Immunofluorescence}

The cells were fixed in 4\% paraformaldehyde, rinsed with $1 \times$ phosphate-buffered saline (PBS) and incubated with $3 \%$ bovine serum albumin (BSA) blocking solution for $60 \mathrm{~min}$ on a shaker. Runx 2 antibody (1:500 dilution) was added directly to the blocking solution and the samples were further incubated for $3 \mathrm{~h}$ at room temperature. The samples were washed with $1 \times$ PBS and incubated with $5 \mu \mathrm{L}$ AlexaFluor ${ }^{\circledR}$ 488-labeled goat anti-rabbit secondary antibody $(2 \mathrm{mg} / \mathrm{mL}$; Molecular Probes, Carlsbad, USA) in $1 \times$ PBS at room temperature, protected from light. After $60 \mathrm{~min}$ of incubation, the cells were washed with $1 \times$ PBS for 3 times of 5 min each. 4,6-diamidino-2-phenylindole (DAPI) was added to PBS for nuclear staining at $1 \times 5000$, followed by washing by PBS. Images were taken with an Olympus IX70 inverted microscope (Olympus, Tokyo, Japan) and processed, using ImageJ software (National Institutes of Health, Bethesda, USA).

\section{Western blot}

After the cajanine treatment, the cells were collected and the harvested proteins were first separated by $10 \%$ sodium dodecyl sulfate-polyacrylamide gel electrophoresis (SDSPAGE), and then transferred to nitrocellulose membranes (Bio-Rad Laboratories, Hercules, USA). The membranes were blocked with 5\% non-fat milk and incubated with primary antibodies at a dilution of 1:1000. The membranes were subsequently incubated with a horseradish peroxidase secondary antibody (Sigma-Aldrich). The protein complex was detected, using enhanced chemiluminescence reagents (Pierce Biochnology Inc., Rockford, USA). Endogenous $\alpha$-tubulin was used as the internal control.

\section{Statistical analysis}

Statistical analyses were performed with Student's t-test or one-way analysis of variance (ANOVA), using Prism v. 5.0 (GraphPad Software, La Jolla, USA).

\section{Results}

\section{Cajanine promotes the osteogenic differentiation of bone marrow mesenchymal stem cells}

It has been well recognized that the human bone marrow stroma contains multipotent mesenchymal cells that give rise to adipocytes and osteoblasts, as well as many other lineages. ${ }^{9}$ To assess the roles of cajanine in the differentiation of BMSCs, we started to investigate whether cajanine isolated from the plant Cajanus cajan L. Millsp. could regulate the osteogenesis of BMSCs in vitro. Bone marrow MSCs were isolated and cultured with or without cajanine for $24 \mathrm{~h}$, then the cells were cultured in regular osteogenic differentiation medium for 16 days. Interestingly, our results show that BMSCs treated with cajanine achieved dramatic increases in extracellular calcium deposits (Fig. 1). Consistently, the results described above were confirmed by Von Kossa staining, which is used for histological visualization of calcium deposits. After 3 weeks of osteogenic induction, the cajanine-treated BMSCs displayed higher

Table 1. The proliferation rates for BMSCs increased by cajanine treatments, measured using an MTT assay

\begin{tabular}{|l|c|c|c|c|c|}
\multirow{2}{*}{\multicolumn{1}{c|}{ Group }} & \multicolumn{5}{|c}{ MTT assay/OD $570 \mathrm{~nm}$} \\
\cline { 2 - 6 } & 3 days & 6 days & 9 days & 12 days & 15 days \\
\hline Control & $0.13 \pm 0.01$ & $0.21 \pm 0.02$ & $0.27 \pm 0.02$ & $0.34 \pm 0.01$ & $0.39 \pm 0.03$ \\
$1 \times 10^{9} \mathrm{~g} / \mathrm{mL}$ & $0.20 \pm 0.02$ & $0.48 \pm 0.01$ & $0.67 \pm 0.01$ & $0.72 \pm 0.01$ & $0.81 \pm 0.01$ \\
\hline $1 \times 10^{8} \mathrm{~g} / \mathrm{mL}$ & $0.21 \pm 0.01$ & $0.51 \pm 0.04$ & $0.73 \pm 0.03$ & $0.80 \pm 0.02$ & $0.83 \pm 0.02$ \\
$1 \times 10^{7} \mathrm{~g} / \mathrm{mL}$ & $0.21 \pm 0.03$ & $0.52 \pm 0.01$ & $0.75 \pm 0.01$ & $0.81 \pm 0.04$ & $0.83 \pm 0.03$ \\
\hline
\end{tabular}

The data is presented as means \pm standard deviations (SD); BMSCs - bone marrow mesenchymal stem cells; MTT - methylthiazol tetrazolium; OD - optical density. 
levels of calcine deposit than the control group (Fig. 2). Taken together, we found that cajanine promoted the osteogenic differentiation of BMSCs.

\section{The effects of in vitro cajanine treatment on the expressions of osteogenesis markers}

To investigate the mechanisms of the cajanine-stimulated osteogenesis of BMSCs, we assessed the osteogenic markers. It has been reported that the expressions of osteopontin (OPN) and type I collagen are the markers of osteogenesis. ${ }^{9}$ As we expected, BMSCs treated with cajanine showed upregulated expressions of OPN and collage I at protein and mRNA levels (Fig. 3A-D). Moreover, Runx2, a transcription factor required for bone formation and the differentiation of BMSCs into osteoblasts, is primarily controlled by the activation of Runx $2 .{ }^{16}$ As we expected, cajanine promoted the nuclear translocation of Runx2 in BMSCs (Fig. 3E), suggesting that the cajanine-activated BMSCs had higher Runx2 activity.

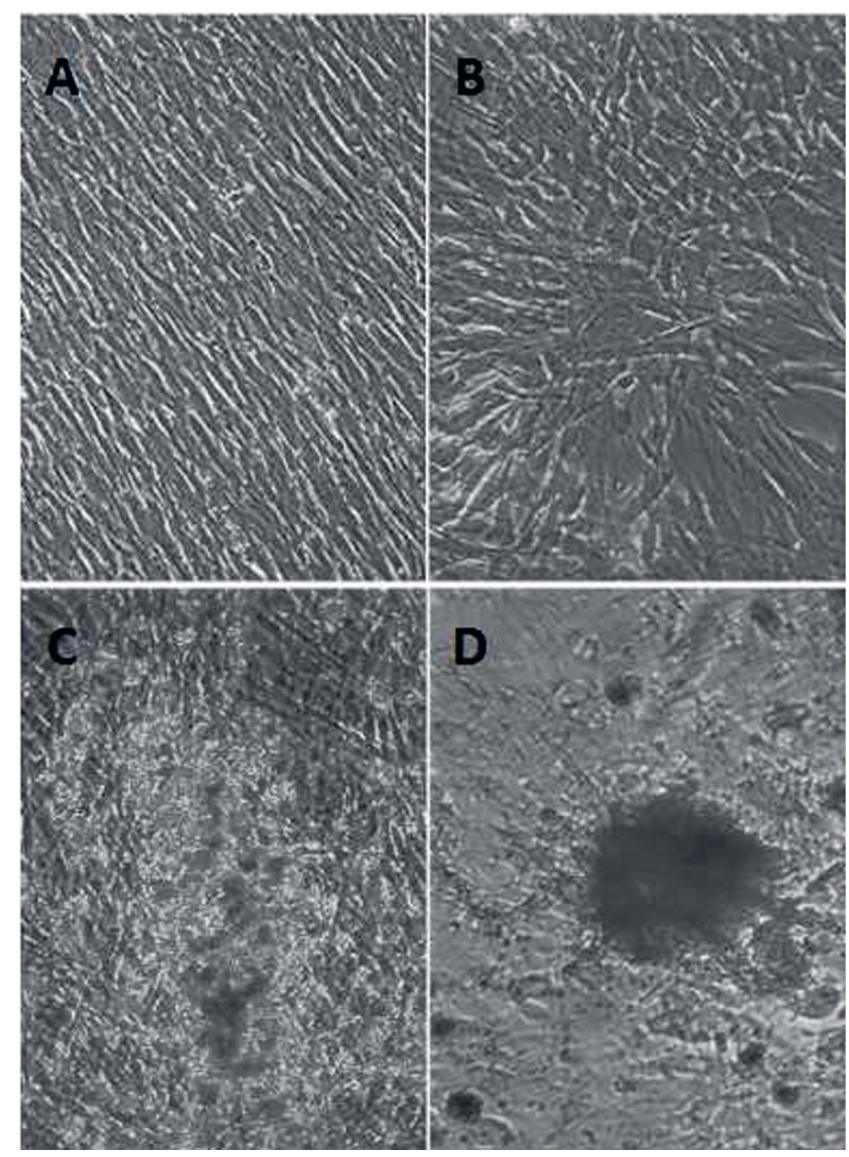

Fig. 1. Cajanine promotes osteogenic differentiation of BMSCs

A - BMSCs treated with PBS as a control; B - BMSCs treated with cajanine for $24 \mathrm{~h} ; \mathrm{C}$ - cells from $\mathrm{A}$ incubated in osteogenic induction medium for 2 weeks; D - cells from B incubated in osteogenic induction medium for 2 weeks.

BMSCs - bone marrow mesenchymal stem cells; PBS - phosphatebuffered saline.

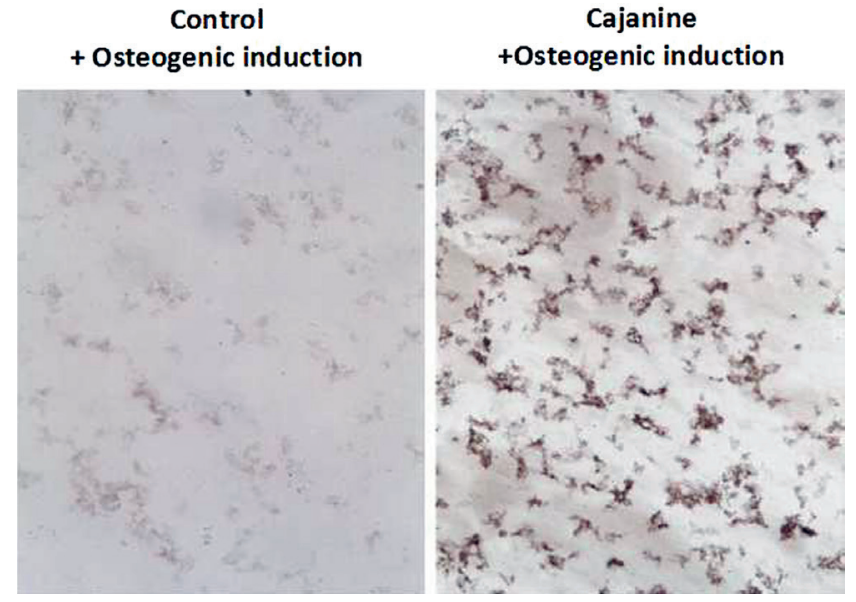

Fig. 2. Von Kossa staining of BMSCs with or without cajanine treatment under the osteogenic induction conditions

A - BMSCs treated with PBS for $24 \mathrm{~h}$ before osteogenic induction; $\mathrm{B}$ - BMSC s treated with cajanine for $24 \mathrm{~h}$, and then incubated with osteogenic induction medium for 2 weeks.

BMSCs - bone marrow mesenchymal stem cells; PBS - phosphatebuffered saline.

\section{The proliferation rate of bone marrow mesenchymal stem cells is stimulated by cajanine}

We next investigated the effects of cajanine on the proliferation of BMSCs in vitro. Bone marrow MSCs were left untreated or were treated with cajanine at 1, 10 or $100 \mathrm{ng} / \mathrm{mL}$ for 3, 6, 9, 12, and 15 days. The MTT assays were performed to measure the cell proliferation ratio. Our data in Table 1 and Fig. 4 demonstrate that cajanine treatment at low concentrations $(1 \mathrm{ng} / \mathrm{mL})$ could significantly increase the cell proliferation after 6 days. At higher concentrations (10 and $100 \mathrm{ng} / \mathrm{mL}$ ), the cell proliferation rates were similar as in the case of low concentrations. Taken together, our results revealed that cajanine promoted both the osteogenic differentiation and proliferation of BMSCs, suggesting cajanine might contribute to the development of therapeutic agents for the clinical applications of tissue engineering.

\section{Cajanine activates cell cycle regulators of bone marrow mesenchymal stem cells}

Our abovementioned results demonstrated that cajanine promotes the proliferation of BMSCs in vitro. We then assessed the cell cycle markers of BMSCs in response to cajanine treatment. To determine the cell cycle consequences of cajanine, we analyzed cell cycle regulators in cajaninetreated BMSCs. Interestingly, our data showed that the phosphorylation of retinoblastoma $(\mathrm{Rb})$ is responsible for a major G1 checkpoint, which was increased by cajanine stimulation, suggesting that cajanine might contribute to the $\mathrm{G} 1$ to $\mathrm{S}$ phase transition (Fig. 5). In addition, we found that the expressions of CDK2, cyclin B1 and CDC 25A\&B 
A

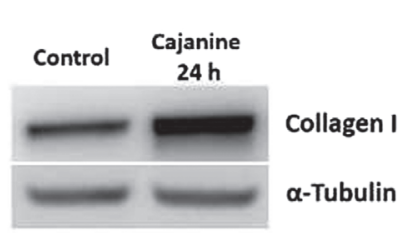

C

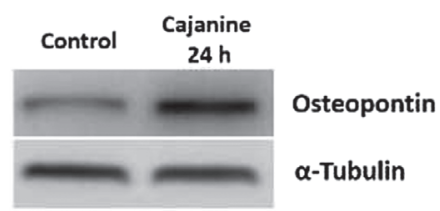

$\mathbf{E}$

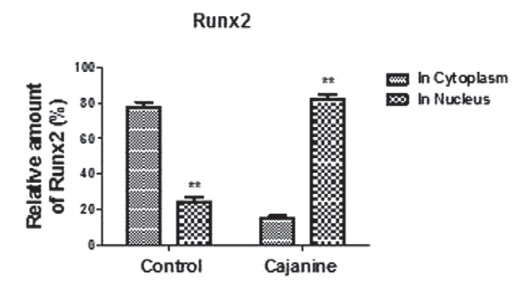

hBMSCs

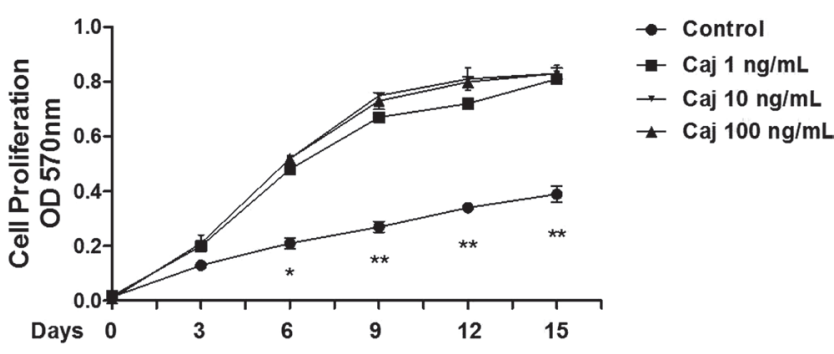

Fig. 4. Cajanine promotes BMSCs proliferation

Bone marrow mesenchymal stem cells treated with a control solution, 1,10 or $100 \mathrm{ng} / \mathrm{mL}$ of cajanine for $3,6,9,12$, and 15 days; the cell proliferation rates measured using a MTT assay.

BMSCs - bone marrow mesenchymal stem cells; MTT - methylthiazol tetrazolium.

were upregulated by cajanine treatment (Fig. 5), which shows that cajanine promoted the proliferation of BMSCs through the modulation of cell cycle progressions.

\section{Discussion}

Cajanus cajan L. Millsp. is a traditional Chinese medicine herb involved in diverse biological processes. In this study, we explored the roles of cajanine in the differentiation and proliferation of hBMSCs. We found that cajanine treatment promoted the osteogenic differentiation of BMSCs in vitro. Since BMSCs are a promising source
Fig. 3. Cajanine treatment upregulates the osteogenic differentiation markers of BMSCs

A - BMSCs left untreated or treated with cajanine for $24 \mathrm{~h}$, then total cell lysates collected and subjected to a western blot analysis, with a-tubulin as a loading control $B$ - BMSCs left untreated or treated with cajanine for $24 \mathrm{~h}$, then the mRNA level of collagen I detected using qRT-PCR; $\mathrm{C}$ - BMSCs left untreated or treated with cajanine for $24 \mathrm{~h}$, then the protein level of OPN detected using western blot with a-tubulin as a loading control;

control D-the mRNA level of OPN detected using qRT-PCR in cajanine-treated or untreated BMSCs.

BMSCs - bone marrow mesenchymal stem cells; qRT-PCR - quantitative reverse transcription polymerase chain reaction; OPN - osteopontin.
B

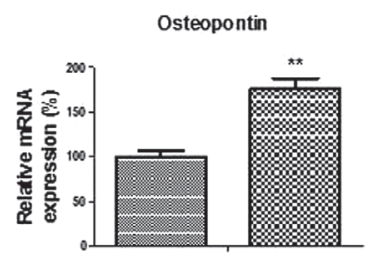

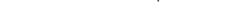


protocols. Moreover, bone differentiation and formation are regulated at the molecular level. Previous studies demonstrated that Runx2 is a transcription factor required for bone formation. ${ }^{17}$ Runx 2 is also a target of bone morphogenetic proteins (BMPs) and is induced by TGF- $\beta$ in pluripotent mesenchymal precursor cells. ${ }^{18}$ Apart from the TGF- $\beta$ pathway, BMSCs osteogenic differentiation was directed through the non-SMAD signaling pathways, mitogen-activated protein kinase (MAPK) signaling and Wnt signaling cascades. ${ }^{19}$ Our results, consistent with the previous studies, showed that the activities of Runx2, as well as other osteogenic markers, are upregulated by cajanine treatment.

Bone marrow MSCs are maintained in a relative state of quiescence until they are activated by a variety of physiological and pathological stimuli. Bone marrow MSCs are capable of proliferating and then differentiating into osteoblasts, chondrocytes, adipocytes, or other mesoderm-type lineages. Therefore, the proliferation of BMSCs is important for maintaining the biological functions of them before differentiation. We report here that cajanine promotes the proliferation of BMSCs by the upregulation of cell cycle regulators. We will continue to explore the detailed mechanisms of the cajanine-stimulated proliferation of BMSCs by measuring other cell proliferation signaling pathways, such as mTOR, Akt or MAPK, in further work. In summary, we discovered a novel function of cajanine in the regulation of osteogenic differentiation and proliferation in BMSCs. Our study will contribute to the cajanine-based drug development for tissue engineering.

\section{References}

1. Nix A, Paull CA, Colgrave M. The flavonoid profile of pigeon pea, Cajanus cajan: A review. Springerplus. 2015;4:125.

2. Duker-Eshun G, Jaroszewski JW, Asomaning WA, Oppong-Boachie F, Brogger CS. Antiplasmodial constituents of Cajanus cajan. Phytother Res. 2004;18(2):128-130.

3. Luo QF, Sun L, Si JY, Chen DH. Hypocholesterolemic effect of stilbenes containing extract-fraction from Cajanus cajan L. on diet-induced hypercholesterolemia in mice. Phytomedicine. 2008;15(11):932-939.
4. Ashidi JS, Houghton PJ, Hylands PJ, Efferth T. Ethnobotanical survey and cytotoxicity testing of plants of south-western Nigeria used to treat cancer, with isolation of cytotoxic constituents from Cajanus cajan Millsp. leaves. J Ethnopharmacol. 2010;128(2):501-512.

5. Luo M, Liu X, Zu Y, et al. Cajanol, a novel anticancer agent from pigeon pea (Cajanus cajan L. Millsp.) roots, induces apoptosis in human breast cancer cells through a ROS-mediated mitochondrial pathway. Chem Biol Interact. 2010;188(1):151-160.

6. Zhang J, Liu C, Sun J, Liu D, Wang P. Effects of water extract of Cajanus cajan leaves on the osteogenic and adipogenic differentiation of mouse primary bone marrow stromal cells and the adipocytic trans-differentiation of mouse primary osteoblasts. Pharm Biol. 2010;48 (1):89-95.

7. Bhargavan B, Gautam AK, Singh D, et al. Methoxylated isoflavones, cajanin and isoformononetin have non-estrogenic bone forming effect via differential mitogen-activated protein kinase (MAPK) signaling. J Cell Biochem. 2009;108(2):388-399.

8. Ye GF, Wang L, Yang RY, Tian RH, Hu YJ, Shen XL. Effects of hydrophobic fraction of Cajanus cajan L. Millsp. on bone density and blood lipid level in obese and diabetic mice. Chinese Pharmacological Bulletin. 2013;29: 961-965.

9. Pontikoglou C, Deschaseaux F, Sensebe L, Papadaki HA. Bone marrow mesenchymal stem cells: Biological properties and their role in hematopoiesis and hematopoietic stem cell transplantation. Stem Cell Rev. 2011;7(3):569-589.

10. Yamasaki S,Mera H, Itokazu M, Hashimoto Y, Wakitani S.Cartilage repair with autologous bone marrow mesenchymal stem cell transplantation: Review of preclinical and clinical studies. Cartilage. 2014;5(4): 196-202.

11. Hu J, Liao H, Ma Z, et al. Focal adhesion kinase signaling mediated the enhancement of osteogenesis of human mesenchymal stem cells induced by extracorporeal shockwave. Sci Rep. 2016;6:20875.

12. Sakaki-Yumoto $M$, Katsuno Y, Derynck R. TGF-beta family signaling in stem cells. Biochim Biophys Acta. 2013;1830(2):2280-2296.

13. Watabe T, Miyazono K. Roles of TGF-beta family signaling in stem cell renewal and differentiation. Cell Res. 2009;19(1):103-115.

14. Ji XY, Xue ST, Zheng GH, et al. Total synthesis of cajanine and its antiproliferative activity against human hepatoma cells. Acta Pharmaceutica Sinica B. 2011;1(2):93-99.

15. Kollmer M, Buhrman JS, Zhang Y, Gemeinhart RA. Markers are shared between adipogenic and osteogenic differentiated mesenchymal stem cells. J Dev Biol Tissue Eng. 2013;5(2):18-25.

16. Gao P, Zhang H, Liu Y, et al. Beta-tricalcium phosphate granules improve osteogenesis in vitro and establish innovative osteo-regenerators for bone tissue engineering in vivo. Sci Rep. 2016;6:23367.

17. Bruderer M, Richards RG, Alini M, Stoddart MJ. Role and regulation of RUNX2 in osteogenesis. Eur Cell Mater. 2014;28:269-286.

18. Chen G, Deng C, Li YP. TGF-beta and BMP signaling in osteoblast differentiation and bone formation. Int J Biol Sci. 2012;8(2):272-288.

19. Rahman MS, Akhtar N, Jamil HM, Banik RS, Asaduzzaman SM. TGF-beta/BMP signaling and other molecular events: Regulation of osteoblastogenesis and bone formation. Bone Res. 2015;3:15005. 